\title{
Evaluation of Postsurgical Stability And Condylar Morphology In Skeletal Class II Patients With Condylar Resorption Following Orthognathic Surgery
}

\author{
Minjiao Wang \\ Shanghai Jiao Tong University School of Medicine \\ Hanjiang Zhao \\ Shanghai Jiao Tong University School of Medicine \\ Xiangyu Wang \\ Shanghai Jiao Tong University School of Medicine \\ Yifeng Qian \\ Shanghai Jiao Tong University School of Medicine \\ Hongbo Yu \\ Shanghai Jiao Tong University School of Medicine \\ Steve GF Shen ( $\square$ maxillofacsurg@163.com ) \\ Shanghai Jiao Tong University School of Medicine
}

\section{Research Article}

Keywords: Skeletal class II malocclusion, orthognathic surgery, condylar resorption, joint spaces

Posted Date: December 30th, 2021

DOI: https://doi.org/10.21203/rs.3.rs-1185890/v1

License: (9) This work is licensed under a Creative Commons Attribution 4.0 International License. Read Full License 


\section{Abstract}

Background: To retrospectively evaluate postsurgical stability and condylar morphology for skeletal class II malocclusion patients with condylar resorption (CR) treated with orthognathic surgery.

Methods: Thirty-five patients treated with combined orthodontic and orthognathic surgery between 2014 and 2018 were enrolled in this retrospective study. CT scans were acquired preoperatively (T0), 2-7 days after surgery ( $T 1$ ), and 1 year postoperatively (T2). The amount of mandibular advancement, postsurgical relapse, condylar morphology and joint spaces were analysed respectively. Statistical analysis was performed using R, version 3.4.3 (R Development Core Team 2010).

Results: The average mandibular advancement and counter-clockwise rotation were $5.51 \mathrm{~mm}$ and -2.82 degrees respectively. The average relapse was $1.08 \mathrm{~mm}$ (19.6\% of the advancement) and 1.13 degrees. The condylar volume showed a postoperative reduction of 161.86 $\mathrm{mm} 3(13.7 \%$ of initial condylar volume). AJS increased after surgery and gradually returned to its original state, while SJS and PJS decreased and remained stable. Surgical advancement of B point was significantly correlated with skeletal relapse. The optimal cut-off values were as follows: MP-FH $\left(40.75^{\circ}\right)$; ramus height $(51.125 \mathrm{~mm})$; SJS $(1.63 \mathrm{~mm})$; surgical displacement $(4.72 \mathrm{~mm})$; CCR $\left(-4.3^{\circ}\right)$; AJSC $(1.07 \mathrm{~mm})$.

Conclusions: Skeletal class II patients with CR have a high risk of postsurgical condylar resorption, which was most related to nonsurgical risk factors such as gender(female) and condylar angle. Condylar had moved posteriorly to the concentric position after surgery and remained quite stable through the 1-year follow-up. Preoperative skeletal patterns such as lower ramus height and surgical procedures such as larger advancement of the mandible were closely associated with sagittal relapse of the mandible.

\section{Background}

Skeletal class II malocclusion patients with receding mandible, high MPA (mandibular plane angle), and open bite are prone to condylar resorption (CR), which may resulted from dysfunctional articular remodelling caused by functional overloading and increased joint friction [1]. For such patients, anterior disc displacement without reduction can be observed by TMJ MRI scans. Loss of cortical bone continuity on condylar head and increased joint space occupied by soft tissue between the condyle and fossa can be observed on CT/CBCT or TMJ MRI scans.

Orthognathic surgery is the main treatment option for severe skeletal class II malocclusion. Lefort I osteotomy and BSSRO for mandible advancement and counter-clockwise rotation of the maxillomandibular complex could dramatically improve facial profile.

However, the stability of mandibular advancement is of vital importance, and several factors may contribute to relapse. The relapse rate of orthognathic surgery for skeletal class II patients ranges from 15-50\% [2-5]. Early postoperative skeletal relapse ( $<6$ months after surgery) is often associated with unstable positioning of the condyle in glenoid fossa and clockwise rotation of the mandible. Long-term postoperative relapse (>1 year) is often related to morphologic changes of the condyle, also known as progressive CR. The suprahyoid soft tissue and the pterygomasseteric sling elongate after surgery, as a result of excessive mechanical loading on the TMJ, which results in postsurgical condylar resorption (PCR).[5-7]However, to the best of our knowledge, the evaluation of postoperative condylar remodelling and joint spaces in patients with pre-existing condylar resorption had rarely been reported.

The aim of this study was to retrospectively evaluate long-term postsurgical outcomes for skeletal class II malocclusion patients with CR who were treated with combined orthodontic and orthognathic surgery. At the same time, we want to explore the susceptible factors of postoperative recurrence in skeletal Class II patients, and then guide the treatment and reduce its recurrence.

\section{Methods}

\section{Patients}

The retrospective study was approved by the hospital ethics committee (No.SH9H-2021-T303-2). All patients were treated with orthognathic surgery for mandibular advancement between 2014 and 2018 at the Department of Oral and Cranio-Maxillofacial Surgery, Shanghai Ninth People's Hospital. The inclusion criteria were as follows: (1) severe class II malocclusion; (2) TMJ MRI before treatment showing condylar bone resorption; (3) treatment with combined orthodontic treatment and orthognathic surgery for mandibular advancement; (4) follow-up for more than 1 year after orthognathic surgery; and (5) availability of pre- and postoperative CT scans. The exclusion criteria were as follows: (1) previous orthognathic surgery or jaw trauma history; (2) severe facial asymmetry or craniofacial syndromes; (3) systemic disease that may contribute to condylar resorption; (4) pregnancy; and (5) incomplete clinical and radiographic records. 
According to the inclusion and exclusion criteria, 35 patients were enrolled in the study: 31 females $(88.6 \%)$ and 4 males (11.4\%). The mean age of the patients was $23.9 \pm 3.6$ years (range 18-33 years). A total of 70 splits of the mandible were performed. Bimaxillary surgery, consisting of a Le Fort I osteotomy and bilateral sagittal split ramus osteotomy (BSSRO), was performed in 33 cases. Single BSSRO were performed in 2 cases. An advanced genioplasty was performed in 23 cases.

\section{Surgical Procedures}

All surgeries were performed under general anesthesia. Virtual surgical planning based on CT data and dental cast was performed in all cases. 3D printed splints and guide plates were fabricated preoperatively. The IMF was released after rigid internal fixation, and dental occlusion was checked. After surgery, the splints were removed from all patients who had fixed orthodontic brackets and stainless steel on their teeth so that patients felt more comfortable.

\section{3d Cephalometry Analysis}

A CT scan of the patient's head was acquired with a slice thickness of $1.25 \mathrm{~mm}$ in the supine position (GE Health care, Fairfield, USA). To address the habitual mandibular protrusion for class II patients, one operator gently guided the mandible backward and upward to seat the condyles in glenoid fossa. Oral silicone rubber for bite recording and temporary occlusion fixation was placed on the occlusal surface of molar teeth. CT data were exported in DICOM format and rendered to 3D models of the facial skeleton using ProPlan Software (ProPlan 2.0, Materialise NV, Leuven, Belgium).

CT scans were acquired for each patient on the following occasions: 2-3 months prior to surgery (T0), 2-7 days after surgery (T1), and at least 1 year after surgery (T2). One calibrated examiner performed all software operations and measurements.

The 3D models of T1 and T2 were superimposed on that of T0 through point and surface registration (Fig. 1A). 3D cephalometric landmarks and planes (Table 1) were used to evaluate mandibular stability and to assess postoperative skeletal relapse, which are analogous to $2 \mathrm{D}$ cephalometric analysis. The distance of B point to $y$-axis (CP: coronal plane) is the main variable for evaluating the sagittal stability of the mandible (Fig. 2).

\section{3d Condylar Morphological Analysis And Joint Space Measurement}

Additionally, 3D ramus morphological analysis and joint space measurement were also performed (Table 1, Measurements 2). Half of the mandible was constructed separately, and the 3D models were superimposed by the maximum surface area overlapping methods on the buccal side of ramus, which is theoretically unchanged in the treatment. (Fig. 1B). Ramus height and condylar angle were measured respectively (Fig. 3A). To determine condylar volume, the mandible was divided by the tangent line parallel to the FH plane through point $\mathrm{C}$, and the superior part was regarded as condylar part, mainly referring to Kim's article[4]. The 3D model of this section was imported into GEOMAGIC Studio 2013 software (Geomagic company, NC, USA) to calculate the condylar volume (Fig. 3B). Linear measurements of joint spaces were performed in accordance with previous study[8], the joint spaces were measured using the centre of each condyle as a reference: (AJS) anterior joint space;(SJS) superior joint space; (PJS) posterior joint spaces (Fig. 3C).

\section{Statistical analysis}

Data were presented as numbers and percentages for categorical variables, and continuous data were expressed as mean \pm standard deviation (SD), unless otherwise specified. Inter-group difference was compared using independent $t$ test and intra-group difference was compared with paired t-tests. We constructed a least absolute shrinkage and selection operator (LASSO) multivariate linear regression model to evaluate the relation between relapse/condylar resorption and risk factors. The final multivariate logistic regression model was built with variables identified under the optimal tuning parameter. The classification performances of MP-FH/ramus height/SJS/surgical displacement/angle of counter-clockwise rotation were evaluated by receiver operating characteristic (ROC) curve. The cut-off probability threshold for each indicator was determined by maximizing the Youden's index.

All the analyses were performed using R, version 3.4.3 (R Development Core Team 2010). A $p$ value $<0.05$ was considered statistically significant.

\section{Results}


The changes (mm or degree) in 3D measurement after orthognathic surgery and long-term follow-up (more than 1 year) for all patients are presented in Table 2. The mean surgical sagittal advancement of point B was $5.51 \pm 3.90 \mathrm{~mm}$, with a vertical downward movement of $1.1 \pm 2.81 \mathrm{~mm}$. The mandible plane angle (MP-FH) decreased $2.82 \pm 3.20^{\circ}$. The mandible body extended $4.81 \pm 2.98 \mathrm{~mm}$ after surgery, which was quite stable during the follow-up period. However, postsurgical relapse (T2-T1) at point B was found in the sagittal $(-1.08 \pm 2.45 \mathrm{~mm})(p=$ $0.014)$ and vertical $(-1.02 \pm 2.03 \mathrm{~mm})(p=0.0053)$ dimensions. The MP-FH showed relapse of $1.13 \pm 2.77^{\circ}(p=0.02)$, which meant clockwise rotation of the mandible.

\section{Condylar morphology and joint spaces}

As shown in Table 3, the ramus height and condylar volumes before surgery were $53.90 \pm 6.86 \mathrm{~mm}^{2}$ and $1183.88 \pm 454.69 \mathrm{~mm}{ }^{3}$, respectively, which were substantially unchanged at T1. The condylar angle increased $2.59 \pm 2.96^{\circ}(p=0.000)$ after surgery, meanwhile, AJS values were significantly higher than those before surgery, while SJS and PJS values were significantly lower, which was consistent with the intraoperative operations of gently pushing the condyle backward and upward to seat the condyles in glenoid fossa. Mean AJS values from initial and follow-up examinations did not differ significantly $(p=0.908)$. However, the follow-up SJS $(p=0.001)$ and PJS $(p=0.032)$ values were still significantly lower than the initial values.

The ramus height and condylar volume at T2 were $52.16 \pm 7.42 \mathrm{~mm}$ and $1022.02 \pm 458.74 \mathrm{~mm}^{3}$. Compared with the values before surgery, the ramus height decreased $1.75 \pm 2.03 \mathrm{~mm}$, and the condylar volume showed a postoperative reduction of $161.86 \pm 192.59 \mathrm{~mm}{ }^{3}$, which were both statistically significant $(p=0.000)$.

\section{Susceptible factors analysis}

Of all 35 patients, 12 patients (34.3\%) displayed more than $2 \mathrm{~mm}$ relapse in the B-CP variable (sagittal dimension). As shown in Table 4, statistically lower initial ramus height $(p=0.029)$, higher surgical displacement $(p=0.000)$ and change of AJS $(p=0.011)$ were demonstrated in patients with significant relapse.

Of all 70 condyles, 40 patients (57.2\%) displayed condylar resorption with more than $10 \%$ of the original volume. As shown in (Additional file 1: Table S1), statistically lower condylar angle $(p=0.022)$ was demonstrated in patients with significant PCR.

Among various preoperative skeletal patterns and surgical procedures, age, ramus height, SJS, surgical displacement, genioplasty and AJSC were screened out and ranked by LASSO multivariate linear regression model. The conclusions were as follows: 1) surgical displacement had the greatest impact on relapse, followed by AJSC and SJS; 2) initial condylar angle had the greatest impact on PCR, followed by MP-FH and gender, as shown in Figs 4 and 5.

The selected data were further analyzed by multivariate logistic regression model. As shown in Additional file 1: Table S2, surgical displacement of B point showed a significant correlation with sagittal skeletal relapse $(p=0.008)$. Moreover, there was a significant correlation between PCR and patient factors such as initial MP-FH (Additional file 1: Table S3, $p=0.04$ ).

ROC curve analysis was performed to determine the value of the susceptible factors for the prediction of long-term relapse. The optimal cut-

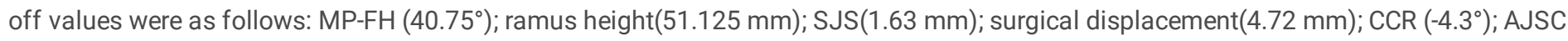
$(1.07 \mathrm{~mm})$ (Additional file 1: Table S4). Patients were classified and analysed according to the cut-off value, which (Table 5) was consistent with Table S4.

\section{Discussion}

Orthognathic surgery is the main option to solve severe dental maxillofacial deformities to obtain an aesthetic profile for skeletal class II malocclusion patients with high MPA. Two jaw surgeries, including Le Fort I osteotomy for maxillary setback and BSSRO for mandibular advancement with counter-clockwise rotation to diminish the occlusion plane and MP-FH, are indispensable to obtain a better functional and aesthetic result[9] .

However, the stability after orthognathic surgery for skeletal class II patients is still in controversy. Previous published studies reported that the relapse rate of mandibular surgical advancement varies considerably from $15-50 \%[3-5,8,10]$. Some studies also emphasized that the procedures typically used for the treatment of Class II patients are quite stable during the first postoperative year. However, these patients may have relevant skeletal changes (more than $2 \mathrm{~mm}$ ) in 1-5 postoperative years $[2,11]$. For patients with condylar resorption, a systematic review performed by Sansare K. et al suggested that the relapse rate was 43\% (ranging from 22-100\%)[12] .

Orthognathic surgery for mandible advancement is a risk factor for overloading of the TMJ $[13,14]$, which leads to condylar dysfunctional remodelling [15]. At the same time, postoperative relapse was closely associated with morphologic changes of the condyles, which was 
known as postoperative condylar resorption (PCR) [16-19]. The incidence of PCR after OS was approximately $2 \%-20 \%[20]$, and the incidence in patients with high MPA was higher[4]. It needs to be noted that late PCR may occur in the first year after surgery, and skeletal relapse gradually aggravate. The common ways of evaluating PCR in previous studies were OPG and lateral cephalograms. As we showed before, the introduction of CT may overcome the shortcomings of X rays and contribute to the use of a validated methodology to measure condylar volume.

Recently, by using CBCT, Xi. et al [4]reported a postoperative decrease in condylar volume of skeletal class II patients after OS, with a mean reduction of $105 \mathrm{~mm}^{3}$ (6.1\% of the original condylar volume). Silva. et al[21]reported significant reductions in superior and medial joint spaces in follow-up examinations and a mean condylar reduction of $57 \mathrm{~mm}^{3}$ after orthognathic surgery in class II patients. However, there is a lack of evaluation of postoperative condylar remodelling and joint spaces in patients with pre-existing condylar absorption.

Since the preponderance of our department is virtual design for orthognathic surgery, the 3D measurement system based on CT data is relatively mature to quantify the surgical changes after surgery. CT scans are not routine for patients' long-term follow-up. For patients with further appeals for removal of titanium plates or facial contouring, CT scans were needed. This is the main source of our long-term 3D CT data, and it is worth noting that most patients rarely need one more CT scan. Therefore, it can be inferred that the patients in this study were less satisfied with the treatment effect or had higher requirements.

The advantages of CT compared to conventional radiographs (orthopantomography and lateral cephalogrammetry) are the possibility of calculating skull models for 3D measurements(linear\angular\volumetric). Overlapping approaches through the anterior skull base and zygomatic bones have been reported in previous studies. However, the overlapping of mandibular ramus was never used to evaluate the change of condylar volume. Considering the rotation of the mandibular ramus after the surgery, the overlapping of mandibular ascending ramus would provide us with a more accurate data.

By reviewing the treatment history of all patients, we found that: all patients underwent preoperative orthodontic procedures (more than 6 months), and the average time between surgical design and operation was 3.4 months, which guaranteed that the condyles were in a stable state at the time of surgery.

In this study, the mean mandibular advancement at point B (B-CP: given that point B is not easily damaged or changed during the surgery) was $5.51 \mathrm{~mm}$. The mean postsurgical relapse after the 1-year follow-up was $1.08 \mathrm{~mm}$ (19.6\% of the surgical displacement), which was comparable with the $19.5 \%$ relapse in previous study[5] (which is in the middle range of that reported in previous studies). Postoperative relapse was acceptable from aesthetic perspectives and all the patients ended with a good relationship of occlusion. The mandible plane angle (MP-FH) decreased $2.82^{\circ}$ after the surgery, which means counter-clockwise rotation of the mandible, with the relapse of $1.13^{\circ} 1$ year after surgery, indicating the occurrence of clockwise rotation of the mandible. Then, patients were further grouped according to the recurrence. Patients suffered with more than $2 \mathrm{~mm}$ relapse showed significantly lower initial ramus height, larger sagittal and vertical displacement at B point, and larger change of AJS.

By measuring the length between the gonion and mental foramen, the following conclusion could be drawn: the mandible body length was elongated and stable by rigid internal fixation. At the same time, a mean reduction of $161.86 \mathrm{~mm}^{3}(13.7 \%$ of initial condylar volume) indicated postsurgical condylar resorption (PCR). AJS increased $0.63 \mathrm{~mm}$ after surgery, while SJS and PJS decreased by $0.29 \mathrm{~mm}$ and $1.43 \mathrm{~mm}$, respectively. This phenomenon is consistent with intraoperative operations of gently pushing the condyle backward and upward to seat the condyles in glenoid fossa. Mean AJS values from initial and follow-up examinations did not differ significantly ( $p=0.908)$. However, the follow-up SJS $(p=0.001)$ and PJS $(p=0.032)$ values were significantly lower than the initial values. These results suggested that a changed anteroposterior condylar position in glenoid fossa, which had moved posteriorly to the concentric position after SSRO with rigid fixation, and remained stable after surgery.

The analysis suggested that patients with a reduction of more than $10 \%$ of the initial volume had a higher MP-FH and lower condylar angle, which was consistent with the study of Ueki. et al[22]. No relationship was observed between the amount of advancement and condylar resorption as demonstrated in previous studies[23], probably due to the presurgical CR status, which was highly vulnerable to PCR.

Finally, to further provide some reference for orthognathic surgical planning, we used (LASSO) multivariate linear regression model and multivariate logistic regression model to assess the association of predictor variables with postoperative relapse.

Among biomechanical factors, the displacement of the mandible had a significant influence on the stability. Large advancement of the mandible could dramatically increase the risk of relapse as a result of stretching of the suprahyoid muscles. [3, 6, 17, 24] For example, patients with mandibular surgical repositioning greater than $8 \mathrm{~mm}$ were found to have a high risk of relapse. [16] Suprahyoid myotomy and Botulin toxin injection into suprahyoid muscles are therapeutic agents to prevent relapse. [25, 26] Additionally, in previous study, the lowest T- 
score and the amount of mandibular advancement exhibited a significant correlation with the relapse tendency.[5] However, no previous study had proposed the specific prognostic factors value of relapse in patients with $\mathrm{CR}$.

In this study, prognostic factors are listed as follows: 1) patient factors(T0): MP-FH, ramus height, condylar volume and initial joint spaces; 2) surgical procedures: fixation type, surgical displacement, counter-clockwise rotation of mandible, changes of condylar angle and joint spaces. Rigid fixation has proven to be more stable than wire osteosynthesis [9], and all patients enrolled in this study were treated with RIF.

Based on the present study, a significant association was found between relapse and preoperative skeletal patterns such as ramus height/SJS, and surgical factors such as surgical displacement at B point/AJSC. And PCR was mainly associated with gender (female), MP$\mathrm{FH}$ and condylar angle, indicating that the baseline status was more relevant and that the presurgical CR status was highly associated with PCR.

Through statistical analysis, those following indicators related to relapse were screened out: ramus height, MP-FH, SJS, the amount of mandibular advancement, Counter-clockwise rotation and AJSC. By using ROC curve analysis, we determined the value of the risk factors above for the prediction of long-term relapse. The optimal cut-off values were as follows: MP-FH $\left(40.75^{\circ}\right)$; ramus height $(51.125 \mathrm{~mm})$; SJS $(1.63 \mathrm{~mm})$; surgical displacement $(4.72 \mathrm{~mm})$; CCR $\left(-4.3^{\circ}\right)$; AJSC $(1.07 \mathrm{~mm})$. Patients with MPA greater than $40.75^{\circ}$, ramus height less than $51.125 \mathrm{~mm}$, the surgical displacement at B point greater than $4.7 \mathrm{~mm}$ and CCR greater than 4.3 degrees should be paid more attention.

The limitation of this study is the lack of MRI evaluations of the TMJ. TMJ MRI examination can clearly show the shape and displacement of articular disc, as well as the size, shape and marrow signal of the condyle, and joint effusion can also be assessed. A follow-up study based on MRI is in progress for further evaluation of postsurgical stability and TMJ status.

\section{Conclusion}

In this study, among 35 patients who showed preoperative condylar resorption, the average mandibular advancement and counter-clockwise rotation were $5.51 \mathrm{~mm}$ and -2.82 degrees, respectively. The average sagittal relapse was $1.08 \mathrm{~mm}$ (19.6\% of the amount of advancement) and 1.13 degrees, which were clinically acceptable. Skeletal characteristics such as lower ramus height and surgical procedures such as larger advancement of mandible increase the risk of skeletal relapse. However, skeletal class II patients with CR have a higher risk of postsurgical condylar resorption. AJS increased after surgery and gradually returned to its original state, while SJS and PJS decreased and remained stable. Future prospective clinical studies on PCR with a larger population and longer follow-up are recommended.

\section{Abbreviations}

CR: condylar resorption PCR: postsurgical condylar resorption

RH: ramus height; CA: condylar angle; CV: condylar volume; CCR: counter-clockwise rotation; AJSC: change of AJS; SJSC: change of SJS; PJSC: change of PJS;

\section{Declarations}

\section{Declaration of competing interest}

None.

\section{Ethical approval and consent to participate}

This study was approved by the Institutional Review Board of Shanghai Ninth People's Hospital, Shanghai Jiao Tong University, School of Medicine (No: SH9H-2021-T303-2) with waiver of informed consent. This study was performed in accordance with the Declaration of Helsinki and its amendments.

\section{Consent for publication}

Not applicable.

\section{Availability of data and materials}

The datasets used and/or analyzed during the current study are available from the corresponding author on reasonable request.

\section{Competing interests}


The authors declare that they have no competing interests.

Funding

The work was supported by the National Key R\&D Program of China (No. 2017YFB1104100), Shanghai Sailing Program (No. 19YF1426500), and the "ChuangKe" Project of Shanghai Ninth People's Hospital (No. CK2019012).

\section{Authors' contributions}

MJW wrote the manuscript. MJW, HJZ, and XYW collected the data, MJW and YFQ conducted the statistical analysis. HBY and GFS modified the paper and designed the study concepts. All authors read and approved the final manuscript.

\section{Acknowledgements}

Not applicable.

\section{References}

1. Wolford LM, Cardenas L: Idiopathic condylar resorption: diagnosis, treatment protocol, and outcomes. Am J Orthod Dentofacial Orthop 1999, 116(6):667-677.

2. Eggensperger N, Smolka K, Luder J, lizuka T: Short- and long-term skeletal relapse after mandibular advancement surgery. Int J Oral Maxillofac Surg 2006, 35(1):36-42.

3. Joss CU, Thüer UW: Stability of the hard and soft tissue profile after mandibular advancement in sagittal split osteotomies: a longitudinal and long-term follow-up study. European journal of orthodontics 2008, 30(1):16-23.

4. Xi T, Schreurs R, van Loon B, de Koning M, Berge S, Hoppenreijs T, Maal T: 3D analysis of condylar remodelling and skeletal relapse following bilateral sagittal split advancement osteotomies. J Craniomaxillofac Surg 2015, 43(4):462-468.

5. Yang HJ, Hwang SJ: Bone mineral density and mandibular advancement as contributing factors for postoperative relapse after orthognathic surgery in patients with preoperative idiopathic condylar resorption: a prospective study with preliminary 1-year follow-up. Oral Surg Oral Med Oral Pathol Oral Radiol 2015, 120(2):112-118.

6. Beukes J, Reyneke JP, Becker PJ: Medial pterygoid muscle and stylomandibular ligament: the effects on postoperative stability. Int J Oral Maxillofac Surg 2013, 42(1):43-48.

7. van der Linden C, van der Linden WJ, Reyneke JP: Skeletal stability following mandibular advancement with and without advancement genioplasty. Int J Oral Maxillofac Surg 2015, 44(5):621-626.

8. Ha N, Hong Y, Qu L, Chung M, Qu R, Cai X, Fang B, Jiang L: Evaluation of post-surgical stability in skeletal class II patients with idiopathic condylar resorption treated with functional splint therapy. J Craniomaxillofac Surg 2020, 48(3):203-210.

9. Frey DR, Hatch JP, Van Sickels JE, Dolce C, Rugh JD: Alteration of the mandibular plane during sagittal split advancement: short- and long-term stability. Oral Surg Oral Med Oral Pathol Oral Radiol Endod 2007, 104(2):160-169.

10. Rocha VA, Neto Al, Rebello IM, de Souza GM, Esteves LS, dos Santos JN, Zanetta-Barbosa D, do Prado CJ: Skeletal stability in orthognathic surgery: evaluation of methods of rigid internal fixation after counterclockwise rotation in patients with class II deformities. Br J Oral Maxillofac Surg 2015, 53(8):730-735.

11. Bailey L, Cevidanes LH, Proffit WR: Stability and predictability of orthognathic surgery. Am J Orthod Dentofacial Orthop 2004, 126(3):273-277.

12. Sansare K, Raghav M, Mallya SM, Karjodkar F: Management-related outcomes and radiographic findings of idiopathic condylar resorption: a systematic review. Int J Oral Maxillofac Surg 2015, 44(2):209-216.

13. Frey DR, Hatch JP, Van Sickels JE, Dolce C, Rugh JD: Effects of surgical mandibular advancement and rotation on signs and symptoms of temporomandibular disorder: a 2-year follow-up study. Am J Orthod Dentofacial Orthop 2008, 133(4):490 e491-498.

14. Kim YI, Jung YH, Cho BH, Kim JR, Kim SS, Son WS, Park SB: The assessment of the short- and long-term changes in the condylar position following sagittal split ramus osteotomy (SSRO) with rigid fixation. J Oral Rehabil 2010, 37(4):262-270.

15. Tanaka E, Detamore MS, Mercuri LG: Degenerative disorders of the temporomandibular joint: etiology, diagnosis, and treatment. Journal of dental research 2008, 87(4):296-307.

16. Hoppenreijs TJ, Freihofer HP, Stoelinga PJ, Tuinzing DB, van't Hof MA: Condylar remodelling and resorption after Le Fort I and bimaxillary osteotomies in patients with anterior open bite. A clinical and radiological study. Int J Oral Maxillofac Surg 1998, 27(2):81-91. 
17. Hwang SJ, Haers PE, Zimmermann A, Oechslin C, Seifert B, Sailer HF: Surgical risk factors for condylar resorption after orthognathic surgery. Oral Surg Oral Med Oral Pathol Oral Radiol Endod 2000, 89(5):542-552.

18. Hwang SJ, Haers PE, Seifert B, Sailer HF: Non-surgical risk factors for condylar resorption after orthognathic surgery. J Craniomaxillofac Surg 2004, 32(2):103-111.

19. Kobayashi T, Izumi N, Kojima T, Sakagami N, Saito I, Saito C: Progressive condylar resorption after mandibular advancement. Br J Oral Maxillofac Surg 2012, 50(2):176-180.

20. Catherine Z, Breton P, Bouletreau P: Condylar resorption after orthognathic surgery: A systematic review. Rev Stomatol Chir Maxillofac Chir Orale 2016, 117(1):3-10.

21. da Silva RJ, Valadares Souza CV, Souza GA, Ambrosano GMB, Freitas DQ, Sant'Ana E, de Oliveira-Santos C: Changes in condylar volume and joint spaces after orthognathic surgery. Int J Oral Maxillofac Surg 2018, 47(4):511-517.

22. Ueki K, Yoshizawa K, Saito Y, Takayama A, Baba N, Kimura Y, Koizumi M, Fujimoto K, Iguchi R, Sato M et al: Evaluation of condylar surface CT values related to condylar height reduction after orthognathic surgery. J Craniomaxillofac Surg 2021, 49(8):639-648.

23. Scheerlinck JP, Stoelinga PJ, Blijdorp PA, Brouns JJ, Nijs ML: Sagittal split advancement osteotomies stabilized with miniplates. A 2-5year follow-up. Int J Oral Maxillofac Surg 1994, 23(3):127-131.

24. Catherine Z, Breton P, Bouletreau P: Management of dentoskeletal deformity due to condylar resorption: literature review. Oral Surg Oral Med Oral Pathol Oral Radiol 2016, 121(2):126-132.

25. Ellis E, 3rd, Carlson DS: Stability two years after mandibular advancement with and without suprahyoid myotomy: an experimental study. Journal of oral and maxillofacial surgery: official journal of the American Association of Oral and Maxillofacial Surgeons 1983, 41(7):426-437.

26. Mucke T, Loffel A, Kanatas A, Karnezi S, Rana M, Fichter A, Haarmann S, Wolff KD, Loeffelbein DJ: Botulinum toxin as a therapeutic agent to prevent relapse in deep bite patients. J Craniomaxillofac Surg 2016, 44(5):584-589.

\section{Tables}

Table1. Definition of the 3D cephalometric landmarks, planes and measurements. 
Landmarks/Plane

\begin{tabular}{|c|c|c|}
\hline \multirow[t]{11}{*}{ Landmarks } & S (sella) & Middle point of the sella turcica \\
\hline & $N$ (nasion) & The intersection of the internasal and frontonasal sutures in the midsagittal plane \\
\hline & Po (porion) & The most upper point on bony external auditory meatus \\
\hline & Or (orbitale) & Lowest point on infraorbital margin of each orbit \\
\hline & B (supramental) & Most posterior midline point on the mandible \\
\hline & Pog(pogonion) & Most anterior midpoint of symphysis of mandible \\
\hline & Me(menton) & Most inferior point on symphysis of mandible \\
\hline & Mf囚mental foramen $\rrbracket$ & The outlet of the mandibular neural tube at the mandibular body \\
\hline & Go (gonion) & Midpoint of posterior border of mandibular angle \\
\hline & Co (condylion) & Exact tip of condyle \\
\hline & C(C-point) & Most caudal point of the sigmoid notch \\
\hline \multirow[t]{4}{*}{ Plane } & $\begin{array}{l}\text { FH (Frankfurt horizontal } \\
\text { plane) }\end{array}$ & A plane defined by the PoL $\backslash$ PoR $\backslash$ OrL \\
\hline & CP (coronal plane) & A plane through $\mathrm{N}$ point, normal to $\mathrm{FH}$ plane and sagittal plane \\
\hline & HP (horizontal plane) & A plane through $\mathrm{N}$ point, parallel to $\mathrm{FH}$ palne \\
\hline & C-plane & A plane through the C-point, parallel to $\mathrm{FH}$ palne \\
\hline \multirow{5}{*}{$\begin{array}{l}\text { Measurement } \\
1\end{array}$} & $\mathrm{~B}-\mathrm{CP} / \mathrm{HP} \otimes \mathrm{mm} \rrbracket$ & The sagittal distance from $\mathrm{B}$ to $\mathrm{CP} / \mathrm{HP}$ \\
\hline & Pog-CP/HP囚mm $\rrbracket$ & The sagittal distance from Pog to $\mathrm{CP} / \mathrm{HP}$ \\
\hline & $\mathrm{Me}-\mathrm{CP} / \mathrm{HP} \nabla \mathrm{mm} \rrbracket$ & The sagittal distance from Me to $\mathrm{CP} / \mathrm{HP}$ \\
\hline & $\mathrm{MP}-\mathrm{FH}\left({ }^{\circ}\right)$ & The angle between MP plane and FH plane \\
\hline & $\begin{array}{l}\text { Mandible body length (Go- } \\
\text { Mf) } \varangle \mathrm{mm} \rrbracket\end{array}$ & The distance from Go to Mf \\
\hline \multirow[t]{7}{*}{$\begin{array}{l}\text { Measurement } \\
2\end{array}$} & $\begin{array}{l}\text { Mandible body length (Go- } \\
\text { Mf) } ₫ \mathrm{~mm} \rrbracket\end{array}$ & The distance from Go to Mf \\
\hline & $\begin{array}{l}\text { Ramus height (Co-Go) } \\
\bigotimes \mathrm{mm} \rrbracket\end{array}$ & The distance from Co to Go \\
\hline & Condylar angle $\left({ }^{\circ}\right)$ & the angle between the $\mathrm{FH}$ plane and condylar line \\
\hline & AJS『mm $\rrbracket$ & $\begin{array}{l}\text { the distance between the anterior-most point on the condyle and the articular fossa, } \\
\text { measured in the sagittal view }\end{array}$ \\
\hline & SJS $₫ \mathrm{~mm} \rrbracket$ & $\begin{array}{l}\text { the distance between the superior-most point on the condyle and the articular fossa, } \\
\text { measured in the sagittal view }\end{array}$ \\
\hline & PJS $₫ \mathrm{~mm} \rrbracket$ & $\begin{array}{l}\text { the distance between the posterior-most point on the condyle and the articular fossa, } \\
\text { measured in the sagittal view }\end{array}$ \\
\hline & Condylar volume $\left(\mathrm{mm}^{3}\right)$ & The volume of condyle \\
\hline
\end{tabular}

Table 2. 3D cephalometric variables analysis between different time points. 


\begin{tabular}{|c|c|c|c|c|c|c|c|}
\hline Variables & T0 & T1 & T2 & T1-T0 & & $\mathrm{T} 2-\mathrm{T} 1$ & \\
\hline & Mean (SD) & Mean (SD) & Mean (SD) & Mean (SD) & $P 1$ value & Mean (SD) & $P 2$ value \\
\hline \multicolumn{8}{|l|}{ Sagittal(mm) } \\
\hline $\mathrm{B}-\mathrm{CP}$ & $-14.67(6.93)$ & $-9.16(5.94)$ & $-10.24(6.09)$ & $5.51(3.90)$ & $<0.0001\left(^{\star}\right)$ & $-1.08(2.45)$ & $0.014(*)$ \\
\hline Pog-CP & $-20.27(8.74)$ & $-10.41(8.22)$ & $-11.66(8.71)$ & $9.86(4.40)$ & $<0.0001\left(^{*}\right)$ & $-1.25(2.94)$ & $0.017\left(^{*}\right)$ \\
\hline Me-CP & $-28.76(9.55)$ & $-18.03(8.87)$ & $-19.23(9.64)$ & $10.73(5.17)$ & $<0.0001\left(^{\star}\right)$ & $-1.2(3.54)$ & 0.053 \\
\hline Mandible body length & $55.36(4.75)$ & $60.16(4.79)$ & $59.90(4.99)$ & $4.81(2.98)$ & $<0.0001\left(^{\star}\right)$ & $-0.26(1.20)$ & 0.07 \\
\hline \multicolumn{8}{|l|}{ Vertical(mm) } \\
\hline $\mathrm{B}-\mathrm{HP}$ & $98.40(5.66)$ & $99.51(5.50)$ & $98.48(4.79)$ & $1.10(2.81)$ & $0.03\left(^{*}\right)$ & $-1.02(2.03)$ & $0.0053(*)$ \\
\hline Pog-HP & $114.36(6.16)$ & $117.15(6.57)$ & $116.17(6.05)$ & 2.78(3.59) & $<0.0001\left(^{*}\right)$ & $-0.98(2.8)$ & $0.045\left(^{*}\right)$ \\
\hline Me-HP & $118.58(6.25)$ & $122.02(6.26)$ & $121.12(5.99)$ & $3.45(3.14)$ & $<0.0001\left(^{*}\right)$ & $-0.90(2.36)$ & $0.03\left(^{*}\right)$ \\
\hline \multicolumn{8}{|l|}{ Angle $\left(^{\circ}\right)$} \\
\hline MP-FH & $40.56(7.87)$ & $37.74(6.32)$ & $38.87(7.05)$ & $-2.82(3.20)$ & $<0.0001\left(^{*}\right)$ & $1.13(2.77)$ & $0.02\left(^{\star}\right)$ \\
\hline
\end{tabular}

Table3. Condylar morphology analysis between different time points.

\begin{tabular}{|c|c|c|c|c|c|c|c|}
\hline Variables & TO & $\mathrm{T} 1$ & $\mathrm{~T} 2$ & T1-T0 & & T2-T0 & \\
\hline & Mean (SD) & Mean (SD) & Mean (SD) & Mean (SD) & $P 1$ value & Mean (SD) & $P 2$ value \\
\hline \multicolumn{8}{|c|}{ Length(mm) } \\
\hline $\begin{array}{l}\text { Ramus } \\
\text { height }\end{array}$ & $53.90(6.86)$ & $53.96(6.82)$ & $52.16(7.42)$ & $0.06(0.83)$ & 0.54 & $-1.75(2.03)$ & $<0.0001(*)$ \\
\hline \multicolumn{8}{|c|}{$\begin{array}{l}\text { Joint } \\
\text { spaces(mm) }\end{array}$} \\
\hline AJS & $1.45(0.52)$ & $2.08(1.04)$ & $1.44(0.55)$ & $0.63(0.97)$ & $<0.0001\left(^{\star}\right)$ & $-0.01(0.51)$ & 0.908 \\
\hline SJS & 1.93(0.85) & $1.64(0.76)$ & $1.59(0.53)$ & $-0.29(0.98)$ & $0.016(*)$ & $-0.34(0.83)$ & $0.001\left(^{*}\right)$ \\
\hline PJS & $2.66(2.29)$ & $1.23(0.71)$ & $2.12(1.14)$ & $-1.43(2.41)$ & $<0.0001\left(^{\star}\right)$ & $-0.53(2.03)$ & $0.032\left(^{*}\right)$ \\
\hline \multicolumn{8}{|l|}{ Angle $\left(^{\circ}\right)$} \\
\hline $\begin{array}{l}\text { Condylar } \\
\text { angle }\end{array}$ & 103.59(4.39) & $106.18(4.31)$ & $105.88(5.07)$ & $2.59(2.96)$ & $\left.<0.00011^{(}\right)$ & $2.52(3.70)$ & $<0.0001(*)$ \\
\hline \multicolumn{8}{|l|}{$\begin{array}{l}\text { Volume } \\
\left(\mathrm{mm}^{3}\right)\end{array}$} \\
\hline $\begin{array}{l}\text { Condylar } \\
\text { volume }\end{array}$ & $1183.88(454.69)$ & $1200.62(458.74)$ & $1022.02(459.12)$ & $16.74(103.80)$ & 0.182 & $-161.86(192.59)$ & $<0.0001(*)$ \\
\hline
\end{tabular}

Table4. Statistical analysis of baseline situation and surgical changes in relation to surgical relapse. 


\begin{tabular}{|c|c|c|c|}
\hline \multirow[t]{2}{*}{ Variables } & \multicolumn{2}{|l|}{ Relapse } & \multirow[t]{2}{*}{$P$ value } \\
\hline & Yes(N=24) & $\mathrm{No}(\mathrm{N}=46)$ & \\
\hline \multicolumn{4}{|l|}{ Baseline(T0) } \\
\hline $\mathrm{MP}-\mathrm{FH}\left(^{\circ}\right)$ & $42.79(7.80)$ & $39.40(7.65)$ & 0.086 \\
\hline Ramus height(mm) & $51.44(6.78)$ & $55.19(6.61)$ & $0.029(*)$ \\
\hline Condylar angle $\left(^{\circ}\right)$ & $103.92(5.43)$ & $103.42(3.79)$ & 0.659 \\
\hline Condylar volume $\left(\mathrm{mm}^{3}\right)$ & $1062.44(472.61)$ & $1247.24(436.89)$ & 0.107 \\
\hline AJS(mm) & $1.60(0.64)$ & $1.36(0.43)$ & 0.073 \\
\hline SJS(mm) & $2.21(0.96)$ & $1.80(0.76)$ & 0.057 \\
\hline PJS(mm) & 2.75(1.93) & $2.61(2.48)$ & 0.816 \\
\hline \multicolumn{4}{|l|}{ Surgical procedures(T1-T0) } \\
\hline Sagittal displacement at B point(mm) & $7.70(3.98)$ & $4.37(3.34)$ & $<0.0001\left(^{*}\right)$ \\
\hline Sagittal displacement at Pog point(mm) & $10.76 \otimes 5.02 \bigotimes$ & $9.40 \rrbracket 3.94 \rrbracket$ & 0.218 \\
\hline Sagittal displacement at Me point(mm) & $12.38 \rrbracket 6.68 \otimes \square$ & $9.88 \rrbracket 3.91 \rrbracket$ & 0.052 \\
\hline Vertical displacement at B point(mm) & $0.03(2.14)$ & $1.66(3.01)$ & $0.021\left(^{\star}\right)$ \\
\hline Vertical displacement at Pog point(mm) & $2.41(4.26)$ & $2.98(3.17)$ & 0.524 \\
\hline Vertical displacement at Me point(mm) & $3.22(3.74)$ & $3.56(2.77)$ & 0.667 \\
\hline Counter clockwise rotation $\left({ }^{\circ}\right)$ & $-3.53((4.18)$ & $-2.45(2.48)$ & 0.179 \\
\hline Change of condylar angle $\left(^{\circ}\right)$ & $3.17(3.03)$ & $2.29(2.91)$ & 0.244 \\
\hline Change of AJS(mm) & $0.08(0.64)$ & $-0.05(0.44)$ & $0.011\left(^{*}\right)$ \\
\hline Change of SJS(mm) & $-0.3(0.66)$ & $0.07(0.80)$ & 0.580 \\
\hline Change of PJS(mm) & $0.67(0.94)$ & $1.02(1.33)$ & 0.944 \\
\hline Condylar resorption $\left(\mathrm{mm}^{3}\right)$ & $-198.56(190.79)$ & $-142.71(192.81)$ & 0.252 \\
\hline
\end{tabular}

Table 5. Statistical analysis of relapse in relation to cut-off of risky factors.

\begin{tabular}{|c|c|c|c|}
\hline & & Relapse & \\
\hline & Group(cut-off) & Mean $₫ \mathrm{SD} \rrbracket$ & $P$ value \\
\hline \multirow[t]{2}{*}{ B-CP (mm) } & $>4.72(n=40)$ & $2.31 \llbracket 2.21 \otimes \square$ & \multirow[t]{2}{*}{$<0.0001{ }^{(*)}$} \\
\hline & $<4.72(n=30)$ & $-0.56 \rrbracket 1.65 \rrbracket$ & \\
\hline \multirow[t]{2}{*}{ MP-FH $\left({ }^{\circ}\right)$} & $>40.75(n=38)$ & $2.01(2.56)$ & \multirow[t]{2}{*}{$<0.0001(*)$} \\
\hline & $<40.75(n=32)$ & $-0.02(1.77)$ & \\
\hline \multirow[t]{2}{*}{ AJSC (mm) } & $>1.070(n=19)$ & $2.58(3.00)$ & \multirow[t]{2}{*}{$<0.001\left(^{\star}\right)$} \\
\hline & $<1.070(n=51)$ & $0.51(1.95)$ & \\
\hline \multirow[t]{2}{*}{ SJS (mm) } & $>1.63(n=42)$ & $1.51(2.48)$ & \multirow[t]{2}{*}{0.074} \\
\hline & $<1.63(n=28)$ & $0.44 \llbracket 2.28 \rrbracket$ & \\
\hline \multirow[t]{2}{*}{$\operatorname{CCR}\left({ }^{\circ}\right)$} & $<-4.300(n=22)$ & $3.07 \rrbracket 2.54 \rrbracket$ & \multirow[t]{2}{*}{$<0.0001\left(^{\star}\right)$} \\
\hline & $>-4.300(n=48)$ & $0.17 \rrbracket 1.78 \rrbracket$ & \\
\hline \multirow[t]{2}{*}{$\mathrm{RH}(\mathrm{mm})$} & $<51.125(\mathrm{n}=21)$ & $2.12(3.32)$ & \multirow[t]{2}{*}{$0.019\left(^{*}\right)$} \\
\hline & $>51.125(\mathrm{n}=49)$ & $0.64(1.82)$ & \\
\hline
\end{tabular}


Figures

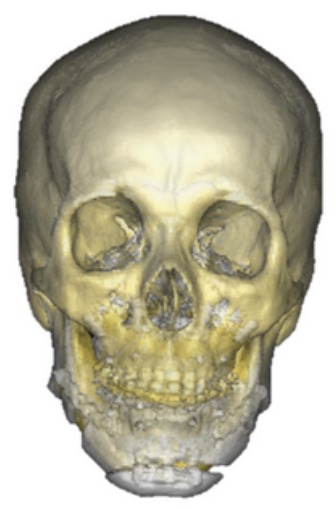

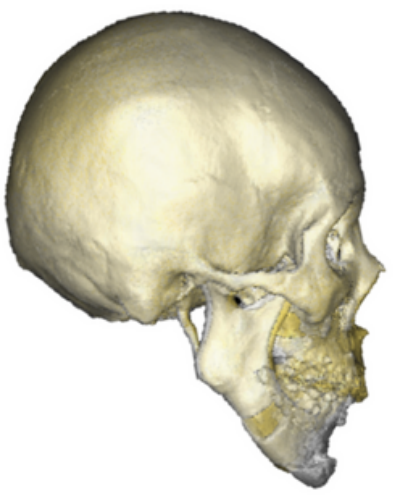

A

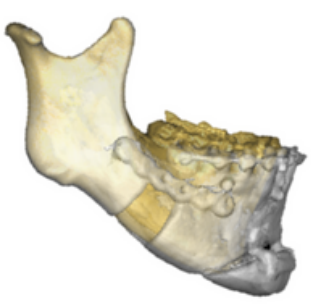

B

\section{Figure 1}

$3 \mathrm{D}$ reconstruction models of different time points were superimposed by the surface-best-fit registration method. (A囚cranial base $\backslash \mathrm{B} \bigotimes$ the proximal part of mandibular ramus)

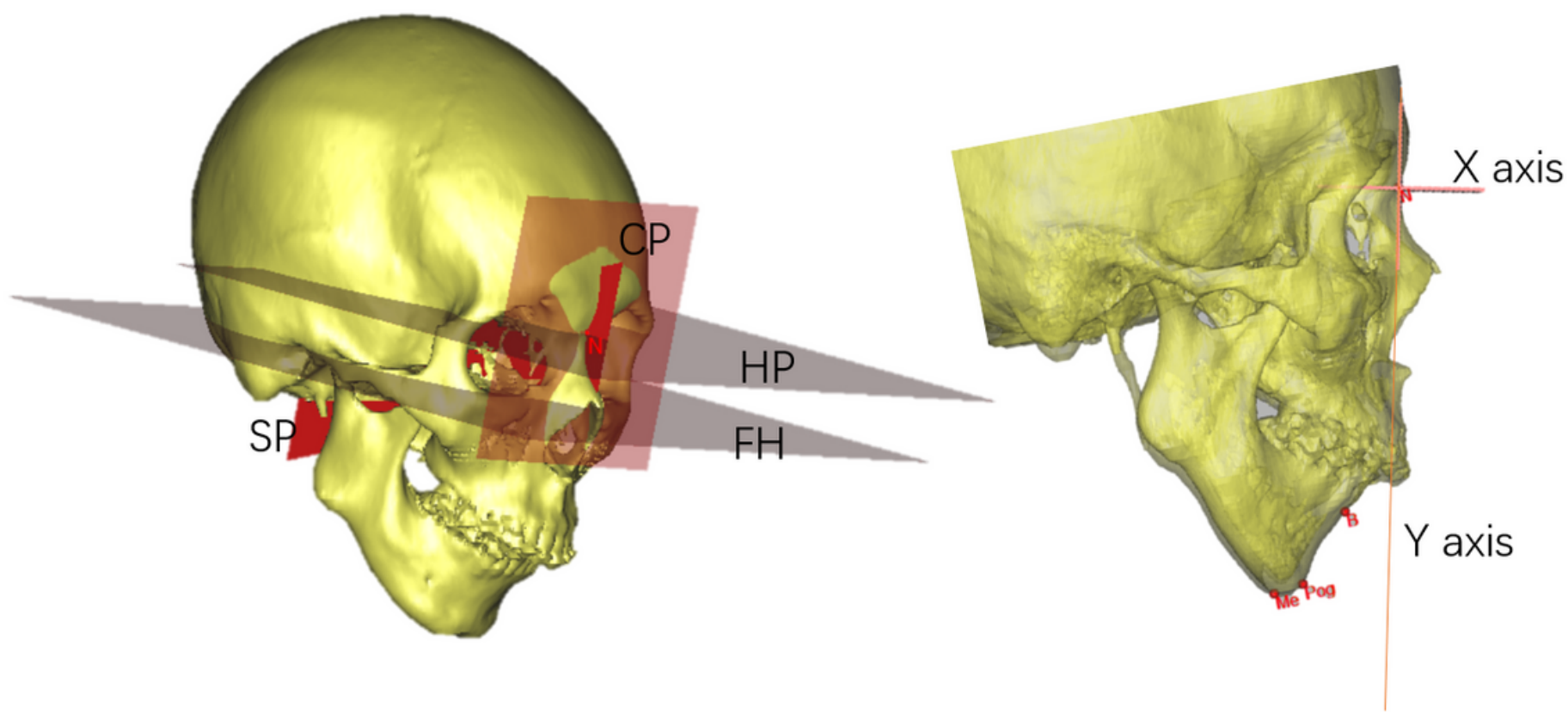

\section{Figure 2}

Anatomic landmarks and planes. oblique view of the set-up of the FH, CP, HP and SP planes. 


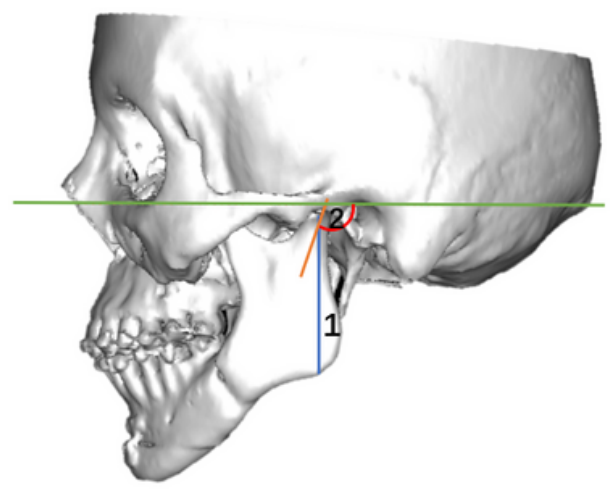

A
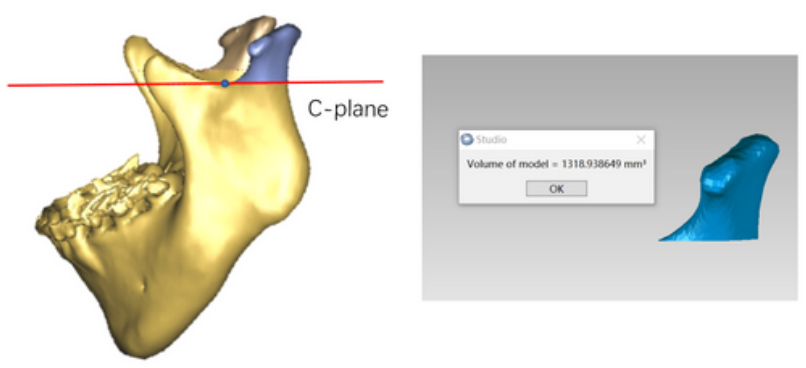

B

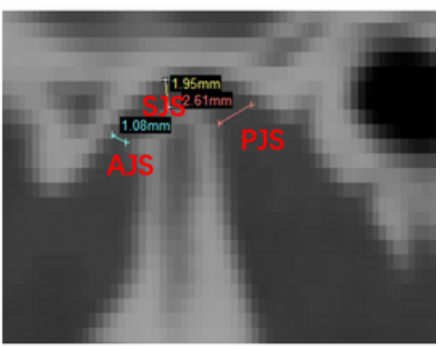

C

\section{Figure 3}

A. Condylar morphological measurement: 1) Ramus height 2) condylar angle

B. Condylar volume: the condylar process that located over the C-plane was segmented and imported into Geomagic Studio software to calculate the volume.

C. Measurement of anterior (AJS), superior (SJS), posterior (PJS) joint spaces in sagittal view of the condyle.

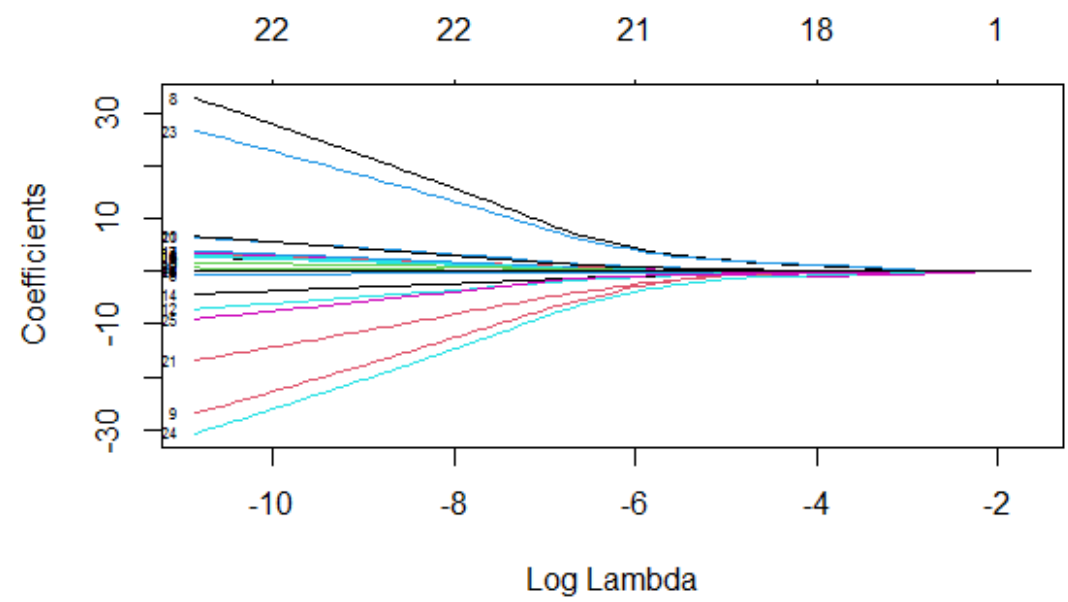

Figure 4

(LASSO) multivariate linear regression model between postoperative relapse and independent parameters 


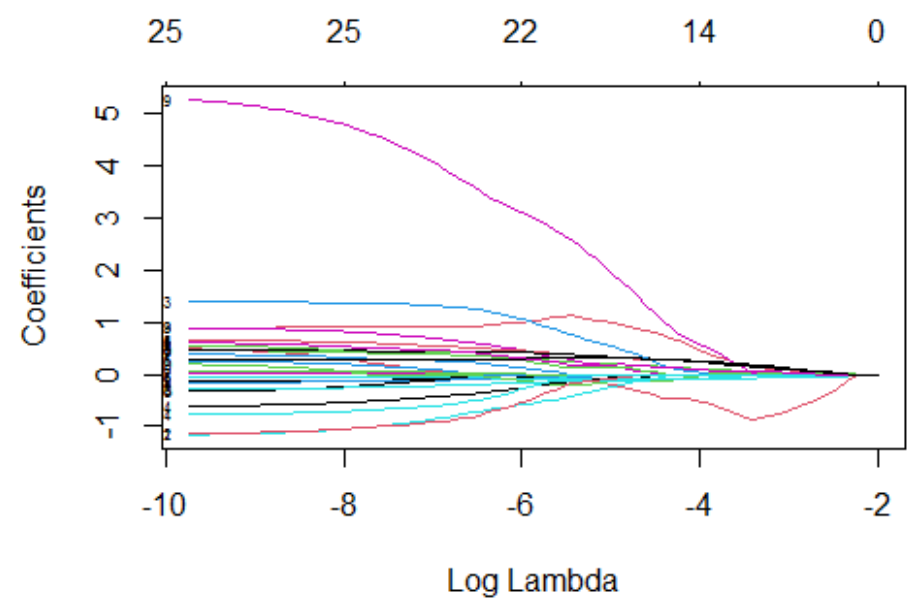

Figure 5

(LASSO) multivariate linear regression model between condylar resorption and independent parameters

\section{Supplementary Files}

This is a list of supplementary files associated with this preprint. Click to download.

- Supplementalinstrument.docx 